\title{
Modification of small bowel mechanosensitivity by intestinal fat
}

\author{
A M Accarino, F Azpiroz, J-R Malagelada
}

\begin{abstract}
Background-Lipids may exacerbate symptoms induced by gut stimuli.

Aim-To determine the mechanism whereby fat exerts this effect.

Subjects-Twenty four healthy subjects were studied during fasting.

Methods-We measured perception (0-6 scale) in response to jejunal balloon distension and transmucosal electrical nerve stimulation; phasic stimuli (one minute) were randomly applied at five minute intervals during intestinal infusion $(2 \mathrm{ml} / \mathrm{min})$ of saline and then Intralipid $2 \mathrm{kcal} / \mathrm{min}$ (high fat; $\mathrm{n}=8$ subjects), Intralipid $0.5 \mathrm{kcal} / \mathrm{min}$ (low fat; $\mathrm{n}=8$ ), or saline $(\mathbf{n}=8)$.

Results-Intestinal lipids increased the perception of jejunal distension regardless of concentration (by $53 \%$ with high fat, $49 \%$ with low fat, and $17 \%$ with saline; p $<0.05$ for both fat loads). This effect could not be attributed to changes in intestinal compliance as intraballoon pressures remained unchanged during lipid infusion ( $2 \%$ change; NS). Sensitisation induced by lipids seemed to be specifically related to intestinal mechanoreceptors because electrical stimulation, which non-specifically activates gut afferents, was perceived equally during saline and lipid administration $(10 \%, 11 \%$, and $15 \%$ change during high fat, low fat, and saline, respectively; NS).

Conclusion-Physiological amounts of lipids heighten intestinal sensitivity by modulating intestinal mechanoreceptor response.

(Gut 2001;48:690-695)
\end{abstract}

4 November 2000

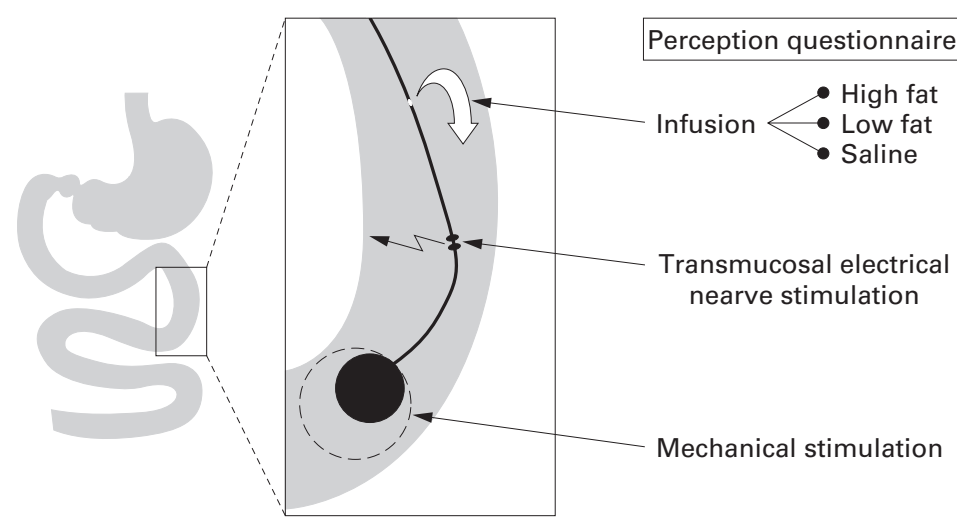

Figure 1 Diagrammatic representation of the probe used. The probe incorporated a perfusion port, a bipolar electrode for electrical stimulation, and an inflatable balloon Electrical stimuli and distensions (one minute duration) were randomly tested (at five minute intervals) during infusion of either saline, low fat, or high fat while measuring intraballoon pressures and perception on a 0-6 scale.
Keywords: intestinal sensitivity; intestinal distension; intestinal electrical nerve stimulation; intestinal afferents; abdominal symptoms

Functional gut symptoms - that is, those without a demonstrable cause- - tend to arise postprandially. However, experimental studies on the mechanisms of gut perception and the genesis of symptoms are usually performed during fasting conditions. ${ }^{12}$ This situation may have inadvertently led to confounding results as meal related factors may modify visceral perception..$^{3-7}$ Following this line of reasoning, we hypothesised that intraluminal nutrients heighten intestinal perception, and our aim was to investigate the potential mechanism involved. Within this framework we considered three putative mechanisms of increased intestinal sensitivity: exaggerated mechanoreceptor response, reduced compliance of the gut wall, and sensory dysfunction of the afferent pathways above receptor level. Hence we investigated the effects of intestinal lipids on: (a) perception of intestinal mechanoreceptor stimulation by balloon distension; (b) intraluminal pressures at different distending volumes - that is, intestinal compliance; and (c) perception of intestinal electrical nerve stimulation. Balloon distension activates perception pathways via stimulation of gut mechanoreceptors whereas transmucosal electrical nerve stimulation produces non-specific activation of afferent pathways and induces perception without relying on any specific receptor. ${ }^{89}$ We previously showed that mechanical and electrical stimuli differentially activate afferent pathways in the gut. ${ }^{9}$ The stimuli were applied during continuous infusion of lipids directly into the jejunum at two different infusion rates: a low rate mimicking intestinal delivery after a low fat meal and a high, supraphysiological rate.

\section{Material and methods \\ PARTICIPANTS}

Twenty four healthy individuals without gastrointestinal symptoms (13 men, 11 women; age range 21-32 years) participated in the study after giving written informed consent. The protocol for the study was previously approved by the Institutional Review Board of the Hospital General Vall d'Hebron.

\section{INTESTINAL STIMULATION}

Intestinal stimulation was performed using a probe previously described in detail. ${ }^{89}$ The probe consisted of a polyvinyl tube assembly (OD $5 \mathrm{~mm}$; ID $3 \mathrm{~mm}$ ) with an inflatable balloon at its distal tip; the probe also featured a bipolar electrode and a perfusion port located $5 \mathrm{~cm}$ and $10 \mathrm{~cm}$ from the tip, respectively (fig 1). 
Phasic distensions were produced by inflating the tip balloon located in the jejunum $15 \mathrm{~cm}$ distal to the angle of Treitz with a predetermined volume of air while recording intraballoon pressure. The balloon was inflated with a syringe by the investigator at a median rate of $10 \mathrm{ml} / \mathrm{s}$ (range $7-13$ ) and at the end of distension the balloon was completely deflated. Intraballoon pressures were recorded by means of pressure transducers (model CDK 3; Cobe, Lakewood, Colorado, USA) on a paper polygraph (model 6006; Letica, Barcelona, Spain). Intraballoon pressures (tonic plus phasic) were averaged during the last half of each distension measured in vitro by injecting air volumes up to $56 \mathrm{ml}$. We used high compliance latex balloons made with condoms; the intrinsic intraballoon pressures showed excellent reproducibility and did not exceed $25 \mathrm{~mm} \mathrm{Hg}$.

Electrical stimulation in the jejunum was produced by a high impedance current stimulator (model 15EO1 Digitism; Dantec, Skovlunde, Denmark) connected to the bipolar electrode $(10 \mathrm{~cm}$ distal to the angle of Treitz) using $0.1 \mathrm{~ms}$ rectangular pulses at a frequency of $15 \mathrm{~Hz}$ with a predetermined intensity. The bipolar electrode was built into the rim of suction holes and when the tube was positioned in the intestine, suction was applied through the central lumen of the tube to ensure firm contact of the electrodes with the gut wall.

PERCEPTION QUESTIONNAIRE

The type and intensity of sensations perceived in response to distension and electrical stimulation of the jejunum were measured using a graded questionnaire. Anatomical questionnaires were also applied to determine the location, extension, and depth of the sensations. After each stimulus, participants were given a signal to report sensations perceived during the preceding one minute period in the questionnaires. These questionnaires have been previously validated and described in detail. ${ }^{2}{ }^{8-13}$

The graded questionnaire included seven visual analogue scales graded from 0 (no perception) to 6 (pain), specifically for scoring: (a) abdominal pressure/fullness, (b) cramp/ colicky sensation, (c) stinging sensation, (d) paraesthesia/flutter-like sensation, (e) warmth, (f) vacuum, and (g) other types of sensation (to be specified). Participants were asked to score any perceived sensation (one or more perceived simultaneously) on the respective scale(s). Perception of somatic stimuli was independently measured by a similar 0-6 scale.

The anatomical questionnaire incorporated a diagram of the abdomen divided into six regions, corresponding to mid and lateral regions above and below the umbilicus. Participants were instructed to mark the location - that is, abdominal region(s) - where the sensations were perceived. To measure the extension of the referral area, we used six numbered circles that respectively covered $0.5 \%$, $2.5 \%, 5 \%, 20 \%, 50 \%$, and $100 \%$ of the abdominal surface in the diagram depicted in the anatomical questionnaire. Participants were asked to select the circle that best represented the extension of the area over which sensations were perceived. To measure depth in the abdomen where sensations were perceived, we used an abdominal wedge diagram depicting four concentric strata from the abdominal surface to the innermost abdominal core, corresponding to depth levels of $0 \%, 33 \%, 66 \%$, and $100 \%$ from the abdominal surface.

To evaluate symptoms induced by fat infusion, we used a specific questionnaire that included the following sensations: sleep, hunger versus satiety, nausea, and feeling of malaise versus well being. Sleep and nausea were scored on a scale from 0 to 6 ; hunger/ satiety was scored on a scale from -6 (hunger) to +6 (satiety). Similarly, malaise and well being were scored on a continuous scale from -6 to +6 . During intestinal infusion of both saline and lipids, the questionnaire was given twice: 10 minutes after starting the infusion and towards the end of the infusion period (four minutes after the first stimulus scored 4 or greater; see experimental design below).

\section{PROCEDURE}

Participants were orally intubated by 8 am after an overnight fast. The tip of the tube was positioned under fluoroscopic control $15 \mathrm{~cm}$ distal to the angle of Treitz so that the stimulating electrode and the perfusion port were located $10 \mathrm{~cm}$ and $5 \mathrm{~cm}$ distal to the angle of Treitz, respectively (fig 1). The studies were conducted in a quiet isolated room with the subjects supine in bed at an angle of $30^{\circ}$ to the horizontal. Distensions and electrical stimuli were tested during continuous infusion (2 $\mathrm{ml} / \mathrm{min}$ ) of saline, Intralipid $0.5 \mathrm{kcal} / \mathrm{min}$ (low fat), or Intralipid $2 \mathrm{kcal} / \mathrm{min}$ (high fat) via the infusion port into the jejunum using an infusion pump (Compact Enteral Feeding Pump model 199235; Sandoz Nutrition Corporation, Minneapolis, Minnesota, USA).The infusion tube and pump were covered so that participants did not know the type of solution being infused.

Ten minutes after intestinal infusion was started, phasic intestinal stimuli of one minute duration were applied in stepwise increments ( $8 \mathrm{ml}$ for distension and $6 \mathrm{~mA}$ for the electrical stimulation) up to the respective threshold for discomfort-that is, perception score of 5 or greater. Individual mechanical and electrical stimuli were applied in random order at five minute intervals. The stimuli were applied blindly, that is, hidden from the participants, and without them knowing either the type or moment the stimulation was being performed. At the end of each stimulus, participants were given the signal to fill out the perception questionnaire as they had been instructed at the beginning of the study.

After the sequence of intestinal stimuli, transcutaneous electrical stimuli of one minute duration were applied in $3 \mathrm{~mA}$ steps at one minutes intervals via skin electrodes on the non-dominant hand, and otherwise using the same technique as for transmucosal electrical stimulation of the jejunum. 
EXPERIMENTAL DESIGN

Each subject participated in only one experiment. In each experiment intestinal stimuli, both mechanical and electrical, as well as somatic stimuli, were tested twice: first during intestinal infusion of saline and subsequently during intestinal infusion of either high fat $(n=8$; five men, three women; age range $22-30$ years), low fat ( $\mathrm{n}=8$; six men, two women; age range $21-32$ years), or saline $(n=8$; two men, six women; age range 20-28 years). The second infusion period was started five minutes after the last stimulus of the first period, and the whole procedure was repeated. In each sequence of stimulation (that is, during the first and second infusion periods) each level of stimulation, including discomfort thresholds, was tested only once. The study was blinded and participants were unaware of the order of the infusions and to which experimental group they were allocated. The effect of fat on sensitivity was measured by comparing the perception scores induced by the same stimuli repeated during the first and second infusion periods (see outcome measures below)

OUTCOME MEASURES

Perception of each stimulus was measured by the score obtained in the graded questionnaire. When more than one abdominal sensation was scored, the highest score was computed for comparisons. In each subject we compared perception of the same levels of stimulation (balloon volumes or electrical intensities) tested during both the first (saline) and second (high fat, low fat, or saline) infusion periods, as we have done previously. ${ }^{11}{ }^{12}$ In each individual stimulus-response trial, the threshold for perception was defined as the smallest volume that induced any kind of perception (perception score $>0$ ), and the discomfort threshold as the smallest volume that induced any type of sensation with score 5 or greater. For each experimental condition tested, stimulusresponse curves were calculated by plotting the perception scores (on the intraballoon pressures) at the three highest levels of stimulation that were repeated during saline and fat infusion. In each subject, the number of times each sensation was scored was counted to calculate the relative frequency (as per cent distribution) of the specific sensations elicited by each type of stimulus under the different conditions tested.

\section{STATISTICAL ANALYSIS}

Mean (SEM) values of the different parameters measured for each type of stimulation under the different conditions tested were calculated. Statistical comparisons of the responses to intestinal distension and electrical stimulation during intestinal fat versus saline infusion were made by the paired Wilcoxon signed rank test using in each subject the cumulative values of the responses to the stimuli repeated during both infusions.

\section{Results}

PERCEPTION OF INTESTINAL DISTENSION

During intestinal infusion of saline, distension of the jejunum induced volume related perception. During intestinal infusion of fat, distension also induced volume related perception but the intensity of perception was significantly higher. Comparing in each subject the cumulative responses to the same volumes repeated during saline and fat infusion, the differences were statistically significant (fig 2) and the effects of low and high fat infusions on gut perception were similar. Thus identical distending volumes repeated in each individual during both saline and fat infusion trials induced $49 \%$ higher perception scores during low fat and $53 \%$ higher scores during high fat infusions $(\mathrm{p}<0.05 v$ saline for both; NS low fat $v$ high fat). The increase in perception was also apparent when comparing thresholds for perception (37 (3) $\mathrm{ml}$ during saline $v 22$ (3) $\mathrm{ml}$ during fat infusion, pooled data for low and high fat; $\mathrm{p}<0.05)$ and discomfort $(48$ (7) $\mathrm{ml}$ during saline $v 38(5) \mathrm{ml}$ during fat infusion, pooled data for low and high fat; $\mathrm{p}<0.05$ ). Duration of infusion was 105 (8) minutes for saline, 80 (7) minutes for low fat, and 71 (8) minutes for high fat; duration of distension sequences was 70 (6) minutes during saline, 41 (7) minutes during low fat, and 45 (8) minutes during high fat infusions $(\mathrm{p}<0.05$ for both low and high fat $v$ saline).

During intestinal infusion of saline, intestinal distensions were perceived as abdominal pressure/fullness (54 (15)\%), colicky sensation (23 $(10) \%)$, or stinging sensation (19 (10)\%). These sensations were largely referred (64 (5) $\%$ of sensations) to an area located on the supraumbilical midline, covering an extension of 19 (4) \% of the abdominal surface, and at a depth of 51 (6)\% beneath the abdominal surface. During fat infusion, the characteristics and quality of perception remained the same. Thus during intestinal fat infusion, intestinal distensions were perceived as abdominal pressure/fullness (47 (8)\%), colicky sensation (25 (10)\%), and stinging sensation (19 (10)\%);

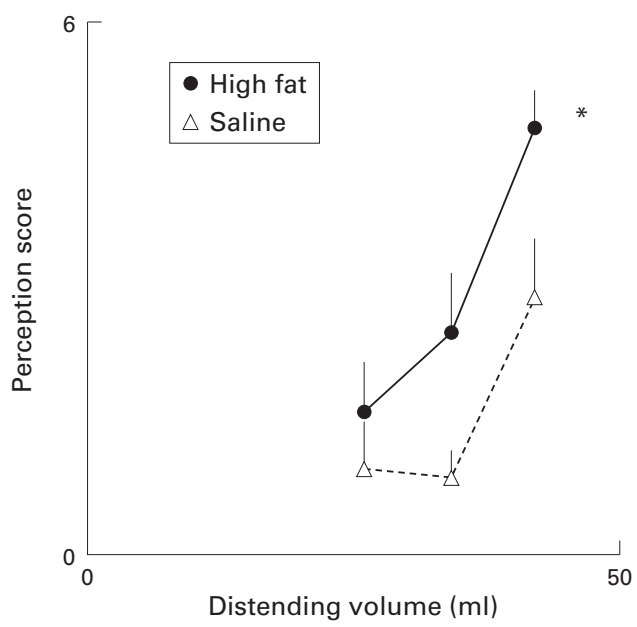

Figure 2 Perception of intestinal distension. Intestinal fat infusion significantly increased perception $\left({ }^{\star} p<0.05\right)$. Data for the three highest stimuli tested in each subject during both saline and high fat infusion are shown. 
$62(10) \%$ of sensations were referred to the supraumbilical midline, more than $14(4) \%$ of the abdominal area, and at a depth of 63 (6)\% beneath the surface (pooled data for high and low fat; NS $v$ saline). It is clear therefore that intestinal fat influenced the intensity but not the quality of perceived abdominal sensations.

INTESTINAL COMPLIANCE

The effects of intestinal fat were unrelated to changes in intestinal compliance as at each distending volume tested intraballoon pressures were similar during saline and fat infusion (fig 3). No difference was found by comparing in each subject the cumulative responses to the same volumes repeated during both saline and fat infusions: at identical distending volumes, intraballoon pressures were $1 \%$ higher during low fat and 3\% higher during high fat infusions (NS $v$ saline). No phasic pressure changes were observed during the distensions with saline or fat infusion.

PERCEPTION OF INTESTINAL ELECTRICAL NERVE STIMULATION

In contrast with the above mentioned effects of fat on perceived distensions, intestinal fat infusion did not modify perception of intestinal nerve stimulation. Transmucosal electrical nerve stimulation induced intensity related perception which was similar during saline, low fat infusion, and high fat infusion (fig 4). No differences were found on comparing in each subject the cumulative responses to the same intensities of stimulation repeated during both saline and fat infusions: perception scores changed by $11 \%$ during low fat infusion and by $10 \%$ during high fat infusion (NS $v$ saline). The lack of effect of intestinal fat was equally patent on comparing thresholds for perception (42 (7) $\mathrm{mA}$ during saline and 41 (6) $\mathrm{mA}$ during fat infusion, pooled data for low and high fat; NS) and thresholds for discomfort (78 (4) $\mathrm{mA}$ during saline and 75 (6) $\mathrm{mA}$ during fat infusion, pooled data for low and high fat; NS).

Abdominal symptoms induced by electrical nerve stimulation of the small bowel were largely similar to those induced by distension:

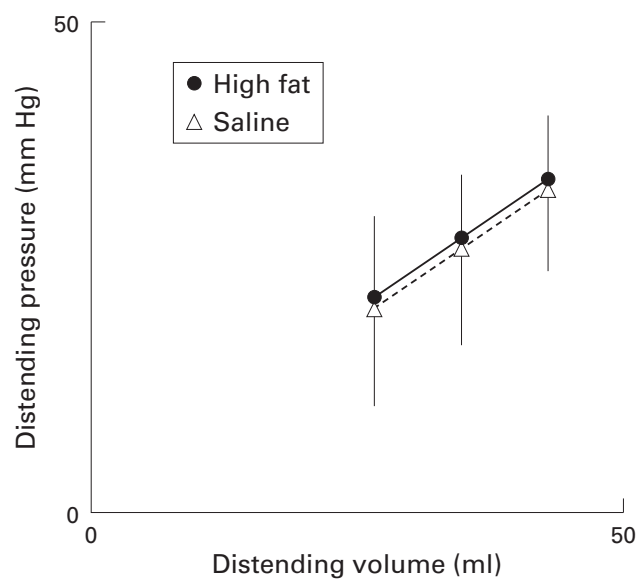

Figure 3 Intestinal compliance. Intestinal fat infusion did not induce any change in intestinal compliance that could explain increased perception of distension. Data for the three highest stimuli tested in each subject during both saline and high fat infusions are shown.

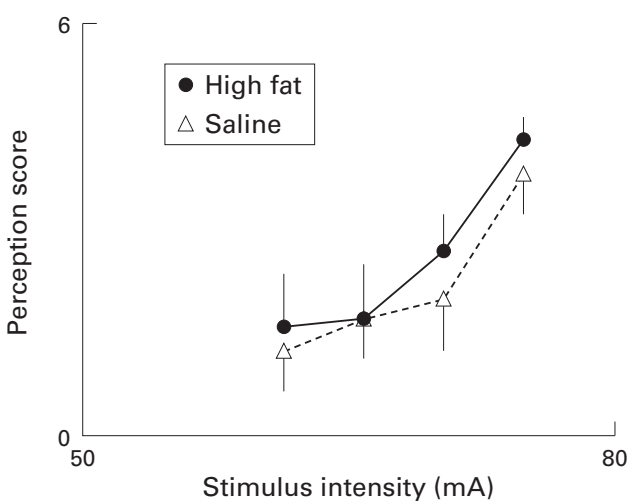

Figure 4 Perception of intestinal electrical nerve stimulation. Intestinal infusion had no effect on perception of transmucosal electrical nerve stimulation. Data for the four highest stimuli tested in each subject during both saline and high fat infusions are shown.

pressure/fullness (34 (11)\%), stinging sensation $(23(8) \%)$, or colicky sensation (18 (7)\%), and only 25 (11)\% of the stimuli induced paresthesia; 59 (11)\% of these sensations were referred to the supraumbilical midline, more than 14 (6)\% of the abdominal area, and at a depth of 51 (5)\% beneath the surface. Intestinal infusion of fat changed neither the type of symptoms (40 (17)\% distension/ fullness, 33 (15)\% stinging sensation, 9 (3)\% colicky sensation, and 18 (11)\% paresthesia) nor the referral pattern (56 (1)\% of sensations referred to the supraumbilical midline, over 15 (5) $\%$ of the abdominal area, and 49 (6) $\%$ beneath the surface; pooled data for high and low fat, NS $v$ saline).

PERCEPTION OF SOMATIC STIMULATION

Intestinal infusion of fat, at high or low loads, had no significant influence on perception of transcutaneous electrical nerve stimulation applied on the hand. When the cumulative responses to the same stimuli repeated during both saline and fat infusions were compared in each subject, perception changed by $-9 \%$ during low fat and by $-3 \%$ during high fat infusions (NS $v$ saline). The discomfort threshold was 52 (6) $\mathrm{mA}$ during saline and 46 (9) $\mathrm{mA}$ during fat infusion (pooled data for high and low fat, NS)

\section{REPRODUCIBILITY STUDIES}

Intestinal distensions and transmucosal electrical nerve stimulation were tested in two consecutive trials during repeat intestinal saline infusion and were found to induce similar perception scores. On retesting, perception of intestinal distension and transmucosal electrical nerve stimulation changed by $17 \%$ and $15 \%$, respectively (NS for both). Likewise, intestinal compliance was similar in the first and second trials (at the same distending volumes the pressures changed by $3 \%$; NS). The type and referral of symptoms induced by intestinal distension and by transmucosal electrical nerve stimulation were also similar in the first and second trials (data not shown). Furthermore, perception of somatic stimuli repeated in the first and second trials during saline infusion changed by $-6 \%$ (NS). Duration of the first and second infusions, as well as 
stimulation sequences, were similar in all instances (data not shown).

EFFECT OF INTESTINAL FAT ON BASAL

PERCEPTION

In the experiments conducted with infusion of either low fat or saline, basal perception was similar to that obtained during the first saline infusion period. In the experiment with the high fat load, basal perception was unchanged after a 10 minute fat infusion but later, when the questionnaire was given the second time (after 83 (13) minutes of high fat infusion), subjects reported nausea and malaise (scores 3.4 (1.0) and 2.5 (1.2), respectively; $\mathrm{p}<0.05 v$ saline).

\section{Discussion}

The major contribution of our study is characterisation of intestinal hypersensitivity induced by luminal lipids. Indeed, we demonstrated that lipids heighten intestinal sensitivity to mechanical, but not electrical, stimulation which suggests that lipids exert a selective modulation on mechanosensitive pathways.

Previous studies have shown that intestinal nutrients, specifically lipids, relax the stomach and increase gastric sensitivity to distension..$^{3-6}$ In the present study, we further characterised the modulatory role of intestinal fat, showing the independence of sensory and motor effects, the widespread sensitisation that affects the small intestine, and its release by very low physiological lipid doses. Furthermore, different putative mechanisms of fat induced hypersensitivity were examined. Firstly, decreased gut compliance was excluded because intraluminal pressures monitored during balloon distension, both tonic and phasic components, were unaffected by intestinal nutrients. Secondly, a non-specific increase in conscious perception was also excluded because it is known that nutrients tend to decrease somatic sensitivity. ${ }^{14}{ }^{15}$ Thirdly, we observed that perception of transmucosal electrical nerve stimulation of the jejunum was not modified by intestinal fat infusion. Transmucosal electrical nerve stimulation may activate various sensory structures in the gut, including terminal endings - that is, receptors and afferent fibres corresponding to both mechanosensitive and mechanoinsensitive pathways-but "in vivo" it does not stimulate intestinal phasic contractions either locally or at adjacent sites. $^{89}$ Hence our study indicates that lipids selectively modulate mechanosensitive pathways. It is important to note that intestinal lipids reproduced sensory dysfunction in patients with the irritable bowel syndrome who have normal or decreased somatic sensitivity ${ }^{916}$ with hypersensitivity to intestinal distension, but not to transmucosal electrical nerve stimulation. ${ }^{9}$

The level of sensitisation of mechanosensitive afferents induced by fat, whether central or peripheral, remains uncertain. Somatosensory physiology assumes that transcutaneous electrical nerve stimulation bypasses the receptor level and directly activates afferent fibres, ${ }^{10} 18$ and by extrapolation, the effects of lipids could be exerted at the mechanoreceptor level.
Support for such a contention can be gained from experimental data showing that cholecystokinin sensitises gut mechanoreceptors $^{61920}$ and that its antagonism reduces fat induced hypersensitivity to gastric distension. ${ }^{6}$ However, the selective sensitisation of mechanosensitive pathways could also have been exerted at higher levels of the central nervous system. For instance, animal experiments have demonstrated central spinovagal interactions, ${ }^{2122}$ and intestinal lipids activate vagal pathways. $^{23} 24$

Several methodological aspects of our study require careful analysis to confirm the reliability of our data. The current literature on visceral sensitivity is confusing and complicated by differences in methodology and lack of definition regarding the nature of mechanoreceptors. Based on different sets of data, the existence of mechanoreceptor subtypes has been suggested, including "in parallel" stretch or elongation receptors, physiologically thought to signal intraluminal volume, "in series" tension receptors activated by both passive distension and active contraction, and mucosal receptors sensitive to friction. ${ }^{2024-26}$ The situation is even more confusing because the responsiveness of mechanoreceptors has been investigated in connection with different afferents, and it has not been well established whether they are linked to reflex regulatory pathways or to splanchnic-spinal pathways signalling conscious perception. Furthermore, data on receptors have been gained from preparations from different regions of the gut, and it is not clear if capacitance organs, such as the stomach and rectum, have the same receptors as conveyer/processor tubular segments, such as the small bowel.

Based on these uncertain data, the stimulation paradigm becomes a key issue in sensitivity studies. In general, distension of the gut involves changes in intraluminal volume, pressure, and wall tension, and these three parameters are interrelated depending on the physical properties of the gut (basically gut compliance). ${ }^{1127}$ However, if gut compliance varies because the region, size of the organ, or motor activity are different, the interrelation of these parameters changes. Under these conditions it is crucial to directly control and standardise the specific parameter that determines in each case the mechanoreceptor response. For instance, gastric distension at a fixed tension level produces similar perception regardless of changing compliance and despite marked differences in intraluminal volumes, ${ }^{11}$ but fixed volume distension results in lower intragastric pressure, wall tension, and perception when compliance increases. ${ }^{112}$ This problem is aggravated in tubular organs where, depending on the compliance and wall resistance to stretch, the distending device may expand longitudinally and stimulate receptors over a longer gut segment. However, the situation is remarkably simplified when the experimental model does not involve differences in gut compliance. In this case, the equation relating volume, pressure, and wall tension remains unchanged, and thus a change in any of these 
parameters results in reproducible changes in the other two. ${ }^{1127}$ Hence in the present experiments, at each distending level, the volumes (infused), intraluminal pressures (measured), wall tension (function of pressure and volume), and conceivably the length of the segment stimulated were all similar during saline and fat infusions, thus supporting the validity of the results regardless of the type of receptors involved.

We undertook a careful experimental design, including all reasonable steps to prevent a response bias. From the different accepted options for testing visceral sensitivity, we chose a paradigm based on comparison of responses to a range of repeated stimuli applied in the same subjects during intestinal infusion of both fat and saline. ${ }^{28}$ Low and high fat infusions were tested in groups with a similar sex distribution, and the study was blinded in that participants did not know the type of infusion they were receiving. The high lipid dose induced basal symptoms but still the effects on perception of gut distension were similar to those observed with the low lipid load that produced virtually no basal sensations. Putative time effects after repeat stimulation were also excluded by reproducibility studies during two consecutive trials of intestinal saline infusion and, furthermore, time effects would also fail to explain the increased perception during fat infusion because the distension sequence was obviously shorter and similar during low and high fat. Hence repeat stimulation over time neither increased mechanical perception nor decreased electrical perception, either of which would have invalidated our results. We applied phasic stimuli of increasing intensity but randomised the order of the individual electrical or mechanical stimuli; a response bias can be reasonably excluded because differential effects were detected (heightened perception of distension but not of electrical stimulation) although both stimuli induced indistinguishable sensations in most tests. Furthermore, the potential influence of the investigator on the subjects was minimised: subjects received standard instructions at the beginning of the study, and after each stimulus were given only a signal to complete the perception questionnaires.

In conclusion, our results provide new information on the characterisation of a viscerosensory modulatory mechanism, and the importance of our study lies in the potential significance of this sensitising mechanism in the genesis of postprandial symptoms in patients with functional gut disorders. ${ }^{2} 32930$

This work was supported in part by the Spanish Ministry of Education (Dirección General de Enseñanza Superior del Ministerio de Educación y Cultura, PM 97-0096). The authors thank Anna Aparici and Maite Casaus for technical support, Gloria Santaliestra for secretarial assistance, and Christine O'Hara for English language editing of the manuscript.
1 Ness TJ, Gebhart GF. Visceral pain: a review of experimental studies. Pain 1990;41:167-86.

2 Azpiroz F. Sensitivity of the stomach and the small bowel: human research and clinical relevance. In: Gebhart GF, ed. Progress in pain research and management, vol 5. Visceral pain. Seattle: IASP, 1995:391-428.

3 Barbera R, Feinle C, Read NW. Nutrient-specific modulation of gastric mechanosensitivity in patients with functional dyspepsia. Dig Dis Sci 1995;40:1636-41.

4 Barbera R, Feinle C, Read NW. Abnormal sensitivity to duodenal lipid infusion in patients with functional dyspepsia. Eur 7 Gastroenrol Hepatol 1995;7:1051-7.

5 Feinle C, Grundy D, Read NW. Effects of duodenal nutrients on sensory and motor responses of the human stomach to distension. Am $\mathcal{F}$ Physiol Gastrointest Liver Physiol 1997;273:G721-6.

6 Feinle C, D'Amato M, Read NW. Cholecystokinin-A receptors modulate gastric sensory and motor responses to gastric distension and duodenal lipid. Gastroenterology 1996; 110:1379-85.

7 Musial F, Crowell MD, Kalveram KT, et al. Nutrient ingestion increases rectal sensitivity in humans. Physiol Behav 1994;55:953-6.

8 Accarino AM, Azpiroz F, Malagelada J-R. Symptomatic responses to stimulation of sensory pathways in the jejunum. Am f Physiol 1992;263:G673-7.

9 Accarino AM, Azpiroz F, Malagelada J-R. Selective dysfunction of mechanosensitive intestinal afferents in the irritable bowel syndrome. Gastroenterology 1995;108:63643.

10 Gracely RH. Studies of pain in normal man. In: Wall PD, Melzack R, eds. Texbook of Pain, 3rd edn. Edinburgh: Churchill Livingstone, 1994:315-36.

11 Distrutti E, Azpiroz F, Soldevilla A, et al. Gastric wall tension determines perception of gastric distension. Gastroenterology 1999;116:1035-42.

12 Notivol R, Coffin B, Azpiroz F, et al. Gastric tone determines the sensitivity of the stomach to distension. Gastroenterology 1995;108:330-6.

13 Iovino P, Azpiroz F, Domingo E, et al. The sympathetic nervous system modulates perception and reflex responses to gut distension in humans. Gastroenterology 1995;108: 636-43.

14 Musial F, Müller J, Kollmannsperger P, et al. Eating induced effects on heart rate, blood pressure, and cutaneous heat effects on heart rate, blood pressure, and cut
pain threshold. Psychol Beiträge 1995;37:32-9.

15 Zmarzty SA, Wells AS, Read NW. The influence of food on pain perception in healthy human volunteers. Physiol Behav 1997; 62:185-91.

16 Cook IJ, Vaan Eeden A, Collins SM. Patients with irritable bowel syndrome have greater pain tolerance than normal subjects. Gastroenterology 1987;93:727-33.

17 Whitehead WE, Holtkotter B, Enck P, et al. Tolerance for rectosigmoid distension in irritable bowel syndrome. Gastroenterology 1990;98:1187-92.

18 Price DD, Long S, Huitt C. Sensory testing of pathophysiological mechanisms of pain in patients with reflex sympaological mechanisms of pain in patients
thetic dystrophy. Pain 1992; 49:163-73.

19 Davison JS, Clarke GD. Mechanical properties and sensitivity to CCK of vagal gastric slowly adapting mechanoreceptors. Am f Physiol Gastrointest Liver Physiol 1988;255;G5561

20 Mayer EA, Gebhart GF. Basic and clinical aspects of visceral hyperalgesia. Gastroenterology 1994;107:271-93.

21 Barber WD, Yuan CS. Gastric vagal-evoked and greater splanchnic-evoked unitary responses in the hypothalamus. Am F Physiol 1993;264:G1133-41.

22 Ren K, Randich A, Gebhart GF. Effects of electrical stimulation of vagal afferents on spinothalamic tract cells in the rat. Pain 1991;44:311-19.

23 Melone J. Vagal receptors sensitive to lipids in the small intestine of the cat. $\mathcal{F}$ Auton Nerv Syst 1986;16:159-70.

24 Sengupta JN, Gebhart GF. Gastrointestinal afferents and sensation. In: Johnson LR, ed. Physiology of the gastrointestinal tract, vol 1, 3rd edn. New York: Raven, 1994:483519.

25 Iggo A. Tension receptors in the stomach and urinary bladder. F Physiol (Lond) 1955;128:593-607.

26 Paintal AS. A study of gastric stretch receptors: their role in the peripheral mechanism of satiation of hunger and thirst. 7 Physiol (Lond) 1954;126:255-70.

27 Fung YC. Biomechanics. Mechanical properties of living tissues. New York: Springer, 1981.

28 Whitehead WE, Delvaux M, and the Working Team. Standardization of barostat procedures for testing smooth muscle ardization of barostat procedures for testing smooth muscle tone and sensory thresholds
Dig Dis Sci 1999;42:223-41.

29 Taggart D, Billington BP. Fatty foods and dyspepsia. Lancet 1966;2:465-6.

30 Houghton LA, Mangall YF, Dwivedi A, et al. Sensitivity to nutrients in patients with non-ulcer dyspepsia. Eur $\mathcal{F}$ Gastroenterol Hepatol 1993;5:109-13. 